\title{
Tecnología romana utilizada en la fabricación de una esquila
}

\author{
J.M. Gómez de Salazar*, M.I. Barrena* y A. Soria*
}

\begin{abstract}
Resumen A principios de los años 70 fueron excavados los restos arqueológicos de una villa tardorromana en la provincia de Palencia. Es conocida como La Olmeda y tiene como mayor atractivo sus mosaicos y los numerosos bienes encontrados. Una de las piezas estudiadas es la denominada esquila o campano de ganado. Se trata de un objeto constituido por hierro y bronce, en su sección hueca y únicamente por hierro en el asa y el badajo. Los estudios de caracterización química, microestructural y de propiedades mecánicas (dureza) han permitido establecer la tecnología utilizada en la fabricación de esta esquila. Este tipo de estudios arqueometalúrgicos permitirán aproximarnos al conocimiento de los procesos tecnológicos llevados a cabo en época romana, en la provincia de Palencia.
\end{abstract}

Palabras clave Arqueometalurgia. Forja. Propiedades mecánicas. Microestructura. Tecnología.

\section{Roman technology used in the manufacture of a cowbell}

\begin{abstract}
At the beginning of the $70^{\text {th }}$ the archaeological artifacts of a roman village in Palencia were excavated. This village was known as La Olmeda and its mosaics and the luxury objects are attractive. The studies are focused on the characterisation of the a cowbell. This object was made with iron and bronze, in his hollow section and with iron in the handle and the clapper. The metallographic characterisation of this piece using optical microscopy, SEMEDX, XRD and hardness test gives information about different fabrication procedure and technological knowledge of the society.
\end{abstract}

Keywords Archaeometallurgy. Forge. Mechanical properties. Microstructure. Technology.

\section{INTRODUCCIÓN}

La villa de La Olmeda se encuentra situada en una llanura en la margen derecha del río Carrión, al oeste de la carretera general que une Palencia y Saldaña ${ }^{[1]}$. Esta villa fue excavada cuando apareció una cama de bocado en los trabajos de explanación, realizados por D. Javier Cortes, hacia el año 1968. La moneda hallada indica la pervivencia de la misma desde el siglo I d. C. hasta el siglo V d. C. ${ }^{[2]}$.

En la época romana, la metalurgia estaba muy desarrollada ${ }^{[3]}$ y se tenían amplios conocimientos en las técnicas de extracción del mineral ${ }^{[4]}$. Además, la fusión y reducción del mismo se realizaba en pequeños hornos donde se introducía este junto con madera y arena, de manera que se daba una fusión y reducción parcial del mineral, y se obtenía una pella que era llevada a un proceso de batida, mediante el cual se conseguían reunir las pequeñas gotas de hierro en un bloque o tocho, al que posteriormente se le daría la forma definitiva ${ }^{[5-7]}$.

\section{MATERIALES Y PROCEDIMIENTO EXPERIMEN- TAL}

El cencerro es una de las piezas encontradas en el patio de la villa de La Olmeda. Se catalogó dentro del grupo de atalajes de caballería, ya que se supone su utilización como cencerro de ganadería. Se trata de una pieza ovalada y hueca en su interior y en cuyo extremo superior (el más estrecho) posee un asa (Fig.1).

El procedimiento experimental seguido consiste en una consolidación de la pieza con metacrilato

$\left({ }^{*}\right.$ Dpto. Ciencia de los Materiales e Ingeniería Metalúrgica. Facultad de Ciencias Químicas. Universidad Complutense de Madrid. 28040 Madrid. 
Tecnología romana utilizada en la fabricación de una esquila J.M. Gómez de Salazar, M.I. BarRena y A. Soria

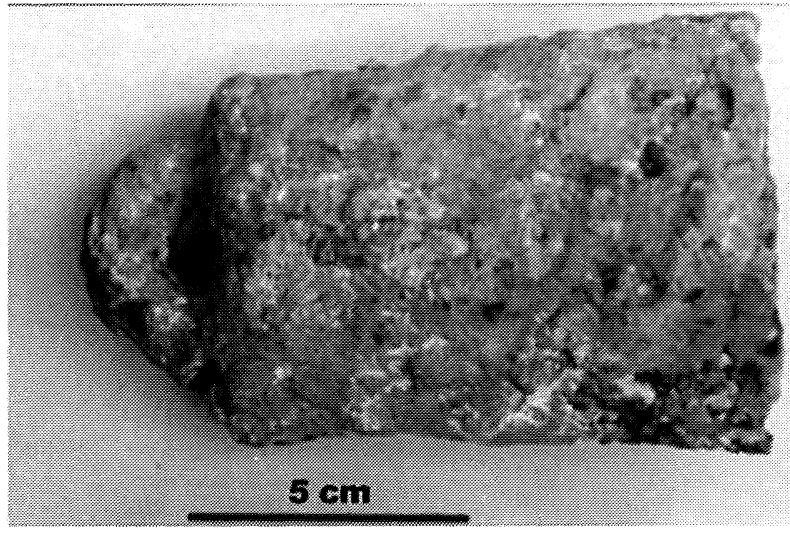

Figura 1. Objeto catalogado como cencerro.

Figure 1. Photography of a cowbell.

diluido en tolueno, para evitar la pérdida de pátinas y óxidos que habían sido analizadas mediante difracción de rayos X (Equipo Philips X'Pert) con anterioridad. La caracterización microestructural se realizó tanto por técnicas de microscopía óptica (banco metalográfico de la marca Reichert $\mathrm{MeF} 3 \mathrm{~A}$ ) como electrónica de barrido (microscopio JEOL JSM-6400 con EDX marca OXFORD). Para ello, se utilizaron técnicas convencionales de preparación metalográfica, donde el ataque metalográfico se realizó con Nital al $2 \%$ y cloruro férrico alcohólico. Se realizó, también, una caracterización mecánica de la pieza utilizando técnicas de medida de dureza con un equipo Akashi AKK-AII.

\section{RESULTADOS Y DISCUSIÓN}

Mediante difracción de rayos X han sido analizadas las tierras que acompañaban esta pieza, así como las pátinas que quedaron adheridas a ella después de su proceso de consolidación. Los picos del difractograma que presentan las tierras quedan identificados como calcita $\left(\mathrm{CaCO}_{3}\right)$ y cuarzo $\left(\mathrm{SiO}_{2}\right)$ (Tabla I). Este último mineral también forma parte de la identificación de los componentes que forman parte de las pátinas, además de hierro $(\mathrm{Fe})$ y cuprita $\left(\mathrm{Cu}_{2} \mathrm{O}\right)$ (Tabla II). El cuarzo y la calcita son componentes habituales de las arenas de la zona donde queda asentado el yacimiento, mientras que la cuprita y el hierro indican la naturaleza de los constituyentes de las pátinas y de la pieza.

Mediante análisis radiográfico (Fig. 2) se aprecia que el asa y el cuerpo del cencerro son dos piezas independientes. Además, en el cuerpo de esta pieza se observa una discontinuidad metálica, desde la zona de asa hasta la base del cencerro, asociada bien a una soldadura longitudinal, bien a la
Tabla I. Análisis de Rayos X de las tierras que acompañan al cencerro

Table I. X-Ray analysis of the cowbell involved lands

\begin{tabular}{|c|c|c|c|c|c|c|c|}
\hline & Difractog & na & & dentificac & ción & & \\
\hline Ángulo & Espaciado & Intensidad & Compuesto & $n^{\circ}$ Ficha & & lanc & \\
\hline $2(\theta)$ & $(\AA ̊)$ & (\%) & & & $\mathrm{h}$ & k & 1 \\
\hline 20,74 & 4,283 & 15,23 & $\mathrm{SiO}_{2}$ & 33-1161 & 1 & & 0 \\
\hline 26,58 & 3,354 & 100 & $\mathrm{SiO}_{2}$ & $33-1161$ & 1 & 0 & 1 \\
\hline 29,30 & 3,048 & 14,63 & $\mathrm{CaCO}_{3}$ & $72-1650$ & 1 & 0 & 4 \\
\hline 36,54 & 2,459 & 11,59 & $\mathrm{SiO}_{2}$ & 33-1161 & 1 & 1 & 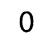 \\
\hline 39,42 & 2,286 & 8,7 & $\mathrm{SiO}_{2}$ & $33-1161$ & 1 & 0 & \\
\hline 40,26 & 2,240 & 5,64 & $\mathrm{SiO}_{2}$ & $33-1161$ & 1 & 1 & 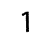 \\
\hline 42,34 & 2,135 & 5,88 & $\mathrm{SiO}_{2}$ & $33-1161$ & 2 & 0 & \\
\hline 43,10 & 2,099 & 3,00 & $\mathrm{CaCO}_{3}$ & $72-1650$ & 2 & 0 & 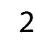 \\
\hline 45,78 & 1,982 & 5,88 & $\mathrm{SiO}_{2}$ & $33-1161$ & 2 & 0 & \\
\hline 47,10 & 1,929 & 3,48 & $\mathrm{CaCO}_{3}$ & $72-1650$ & 0 & 2 & 2 \\
\hline 47,50 & 1,914 & 3,24 & $\mathrm{CaCO}_{3}$ & $72-1650$ & 0 & 1 & $\varepsilon$ \\
\hline 48,46 & 1,878 & 3,24 & $\mathrm{CaCO}_{3}$ & $72-1650$ & 1 & 1 & S \\
\hline 50,10 & 1,821 & 21,7 & $\mathrm{SiO}_{2}$ & 33-1161 & 1 & 1 & 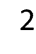 \\
\hline 50,74 & 1,799 & 2,04 & $\mathrm{SiO}_{2}$ & 33-1161 & 0 & 0 & J \\
\hline 54,82 & 1,675 & 8,15 & $\mathrm{SiO}_{2}$ & $33-1161$ & 2 & 0 & 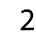 \\
\hline 59,90 & 1544 & 10,07 & $\mathrm{SiO}_{2}$ & $33-1161$ & 2 & 1 & \\
\hline 63,94 & 1,456 & 2,28 & $\mathrm{SiO}_{2}$ & 33-1161 & 1 & 1 & 3 \\
\hline 67,66 & 1,385 & 8,39 & $\mathrm{SiO}_{2}$ & 33-1161 & 2 & 1 & 2 \\
\hline 68,26 & 1,374 & 8,51 & $\mathrm{SiO}_{2}$ & $33-1161$ & 2 & 0 & 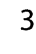 \\
\hline 73,42 & 1,290 & 3,36 & $\mathrm{SiO}_{2}$ & 33-1161 & 1 & 0 & \\
\hline
\end{tabular}

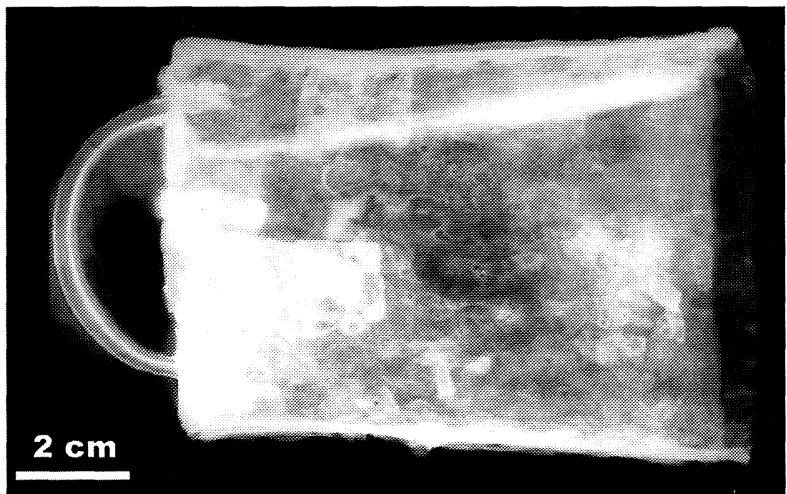

Figura 2. Radiografía frontal del objeto.

Figure 2. Radigraphy of the cowbell in the longitudinal section.

existencia de un badajo. Por último, destaca la presencia de metales de distinta naturaleza manifestada por las láminas de diferente tonalidad, tanto alrededor del cuerpo (transversalmente) como en la 
Tecnología romana utilizada en la fabricación de una esquila J.M. GÓMEz DE SALAzAR, M.I. BARRENA y A. SORIA

Tabla II. Análisis de Rayos $X$ de las pátinas adheridas al cencerro

Table II. X-Ray analysis of cowbell natural patinas

\begin{tabular}{|c|c|c|c|c|c|c|}
\hline & Difractog & ama & & Identificac & ción & \\
\hline Ángulo & Espaci & Intensidad & Compuesto & no Ficha & & anos \\
\hline $2(\theta)$ & $(\AA ̊)$ & (\%) & & & & k I \\
\hline 26,58 & 3,354 & 100 & $\mathrm{SiO}_{2}$ & $33-1161$ & 1 & $\begin{array}{ll}0 & 1\end{array}$ \\
\hline 29,42 & 3,036 & 40,08 & $\mathrm{Cu}_{2} \mathrm{O}$ & $78-2076$ & 1 & 10 \\
\hline 36,42 & 2,467 & 37,89 & $\mathrm{SiO}_{2}$ & $33-1161$ & 1 & 10 \\
\hline 39,38 & 2,288 & 26,98 & $\mathrm{SiO}_{2}$ & $33-1161$ & 1 & 02 \\
\hline 40,22 & 2,242 & 17,86 & $\mathrm{SiO}_{2}$ & $33-1161$ & 1 & 11 \\
\hline 42,62 & 2,121 & 24,80 & $\mathrm{Cu}_{2} \mathrm{O}$ & $78-2076$ & 2 & 00 \\
\hline 44,62 & 2,031 & 74,01 & $\mathrm{Fe}$ & 06-0696 & 1 & 10 \\
\hline 45,70 & 1,985 & 12,50 & $\mathrm{SiO}_{2}$ & 161 & 2 & $\begin{array}{ll}0 & 1\end{array}$ \\
\hline 50,14 & 1,819 & 46,43 & $\mathrm{SiO}_{2}$ & $33-1161$ & 1 & 12 \\
\hline 54,86 & 1,673 & 14,68 & $\mathrm{SiO}_{2}$ & 33-1161 & 2 & 02 \\
\hline 57,74 & 1,597 & 8,13 & $\mathrm{SiO}_{2}$ & $33-1161$ & 2 & 10 \\
\hline 59,90 & 1,544 & 23,01 & $\mathrm{SiO}_{2}$ & $33-1161$ & 2 & 11 \\
\hline 61,26 & 1,513 & 13,69 & $\mathrm{Cu}_{2} \mathrm{O}$ & $78-2076$ & 2 & 20 \\
\hline 64,90 & 1,437 & 8,93 & $\mathrm{Fe}$ & 06-0696 & 2 & 00 \\
\hline 67,66 & 1,385 & 15,08 & $\mathrm{SiO}_{2}$ & $33-1161$ & 2 & 12 \\
\hline 68,06 & 1,378 & 12,69 & $\mathrm{SiO}_{2}$ & $33-1161$ & 2 & 03 \\
\hline 73,46 & 1,281 & 16,67 & $\mathrm{Cu}_{2} \mathrm{O}$ & $78-2076$ & 3 & 11 \\
\hline 75,62 & 1,250 & 3,97 & $\mathrm{SiO}_{2}$ & $33-1161$ & 3 & 02 \\
\hline 80,02 & 1,199 & 5,16 & $\mathrm{SiO}_{2}$ & $33-1161$ & 2 & 21 \\
\hline 81,18 & 1,185 & 7,34 & $\mathrm{SiO}_{2}$ & $33-1161$ & 1 & 14 \\
\hline 82,30 & 1,172 & 75,79 & $\mathrm{Fe}$ & $06-0696$ & 2 & 11 \\
\hline
\end{tabular}

zona superior del mismo, donde esta alojada el asa. Una radiografía lateral (Fig. 3) indica que es posible la soldadura de dos medias campanas en esta zona.

La caracterización microestructural fue realizada en una sección transversal de la zona central, donde se muestra la presencia de dos metales de diferente naturaleza y dispuestos en forma laminar (Fig. 4). Su presencia no es continuada, sino que hay zonas a lo largo de la sección donde una de las láminas ha desaparecido.

Por otro lado, se aprecia una masa metálica adherida en una de las paredes mediante una masa cementante de tierras y que debe ser el badajo de la pieza, el cual presenta una falta de material longitudinal en la zona central de su sección transversal.

La microestructura que presenta el badajo es la de un acero de bajo contenido en carbono donde

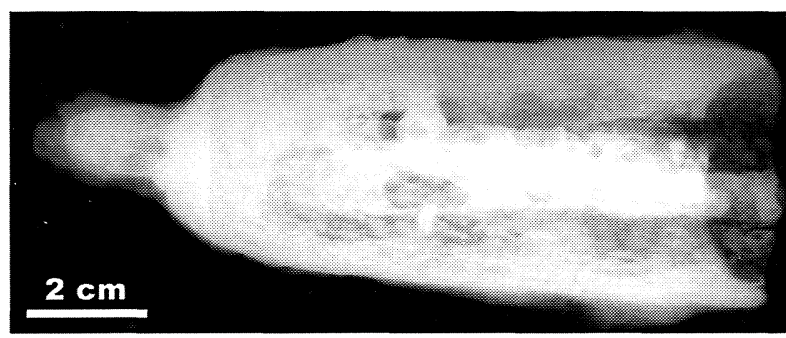

Figura 3. Radiografía lateral del objeto.

Figure 3. Side radiography of the cowbell.

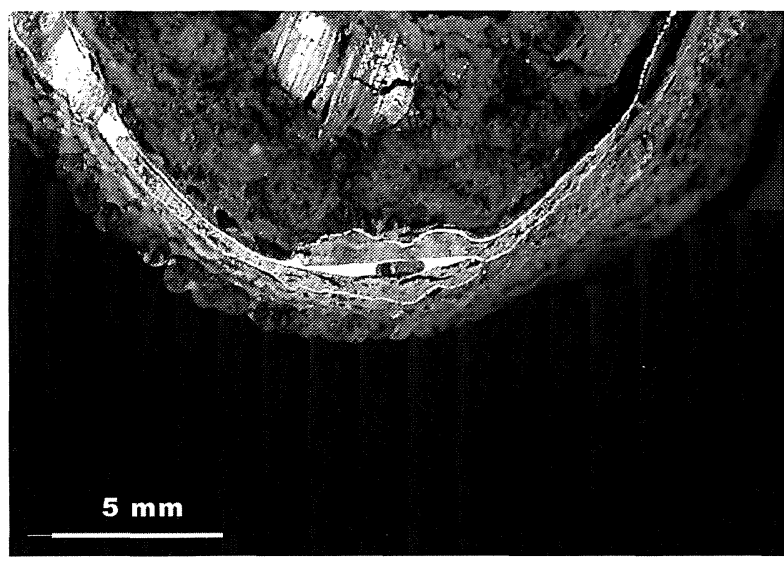

Figura 4. Sección transversal del cencerro.

Figure 4. Transversal section of the cowbell.

las zonas internas poseen una microestructura ferrítica con numerosas escorias y huecos (Fig. 5), mientras que las externas, más carburadas, poseen una microestructura de acero hipoeutectoide ferrítico-perlítico (Fig. 6). No se aprecian indicios de soldadura entre láminas de distinto contenido en carbono, lo que indica que los diferentes

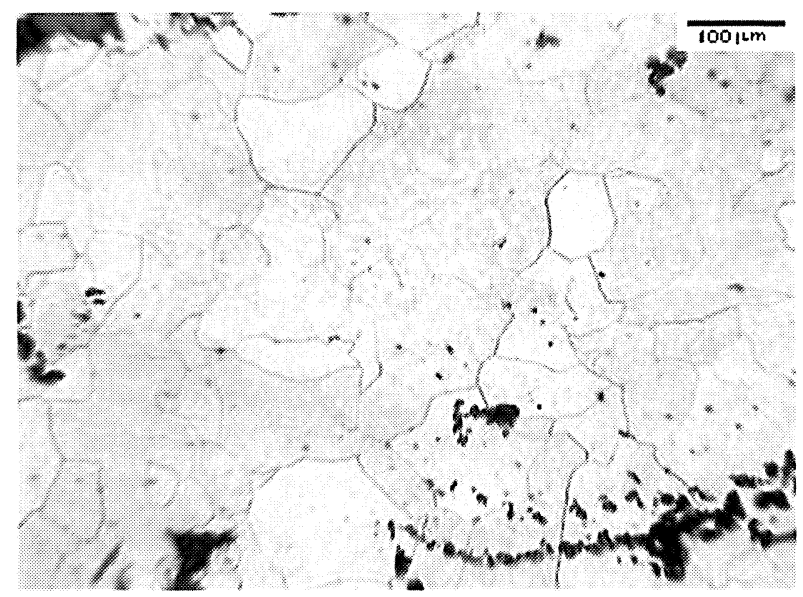

Figura 5. Micrografía de la zona interna del badajo con microestructura de hierro ferrítico.

Figure 5. Clapper internal zone micrography. Ferritic microstructure.

Rev. Metal. Madrid Vol. Extr. (2005) 412-416 


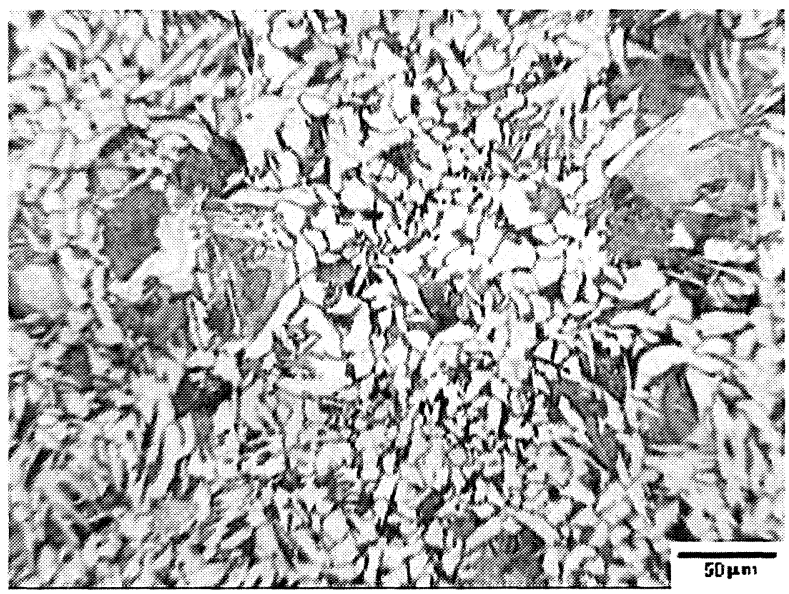

Figura 6. Micrografía de la zona externa del badajo con microestructura ferrítico perlítica.

Figure 6. Clapper external zone micrography. Ferritic-perlitic microstructure.

contenidos en este elemento, presentados en la sección, son debidos a un proceso termoquímico de carburación, por lo que se deduce que el badajo está fabricado de una sola pieza.

El cuerpo del cencerro está compuesto de láminas de metales de distinta naturaleza, que se muestran dispuestas paralelamente (Fig. 7). Una denominada A de hierro con estructura hipoeutectoide (Fig. 8). Otra denominada $\mathrm{C}$ de cobre, hierro y estaño (Fig. 9), que contiene fases globulares de distinta naturaleza. La inclusiones o fases oscuras poseen una naturaleza metálica férrea, mientras que la matriz está formada por bronce de estaño (Tabla III). Las fases globulares de tonos blancos, marcadas como D, están formadas por cobre y plomo

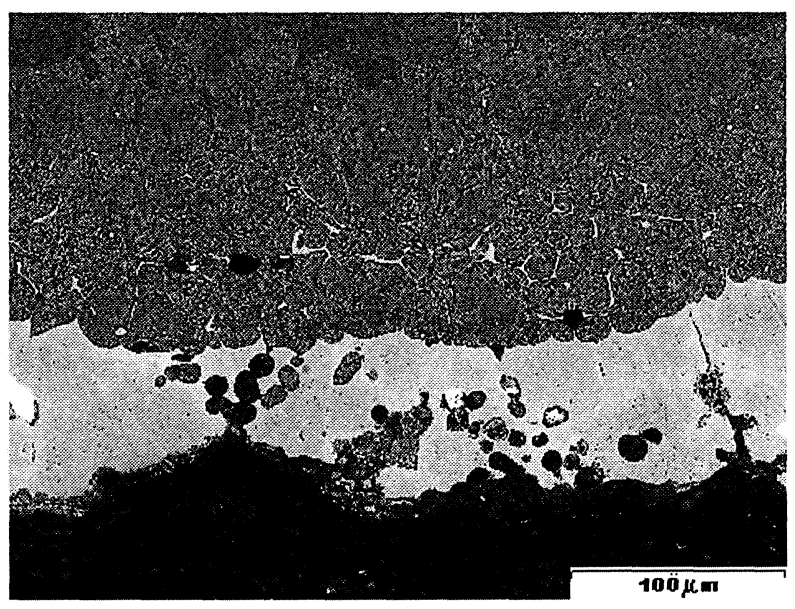

Figura 7. Microestructura del cuerpo del cencerro. Zona de coexistencia de láminas metálicas con diferente naturaleza.

Figure 7. Cowbell microstructure. Coexistence zone of the different metallic sheets.

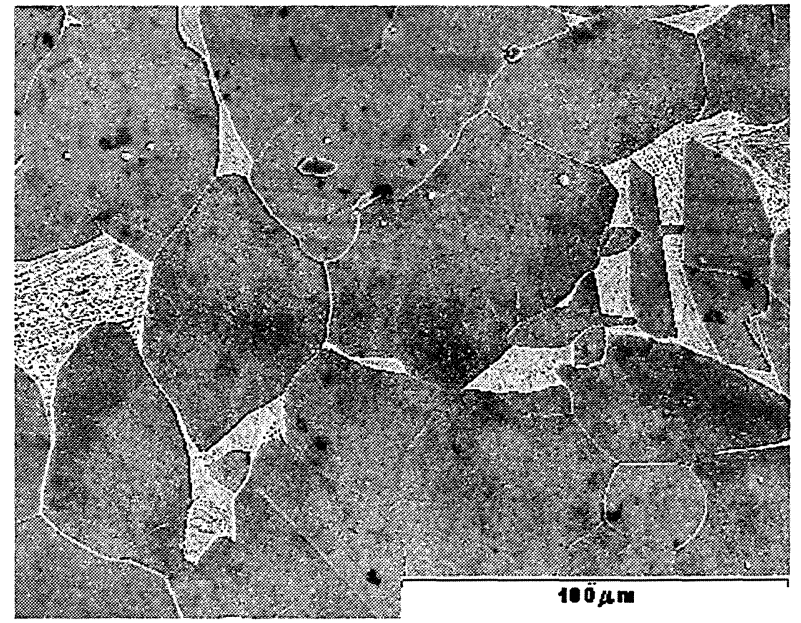

Figura 8. Matriz ferrítico perlítica de acero hipoeutectoide en la zona A de la figura 7.

Figure 8. Ferritic-perlitic matriz of the zone $A$ in the figure 7.

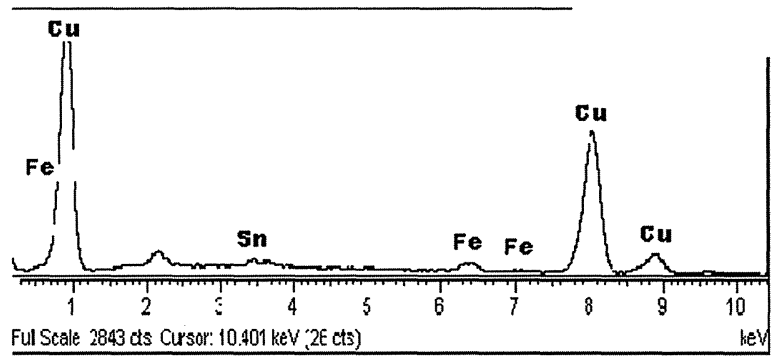

Figura 9. Análisis EDS de la zona C marcada en la figura 7.

Figure 9. EDX Identification of the zone $C$ composition in the figure 7 .

Tabla III. Composiciones de las fases globulares oscuras y matriz presentes en la zona $C$ de la figura 7

Table III. Weight Composition of dark globular phases and matrix present in the zone $C$ of the figure 7

\begin{tabular}{ccc}
\hline \multirow{2}{*}{ Elemento } & \multicolumn{2}{c}{ \% En Peso } \\
\cline { 2 - 3 } & Fases & Matriz \\
\hline $\mathrm{Fe}$ & 81,55 & 2,06 \\
$\mathrm{Cu}$ & 16,57 & 92,87 \\
$\mathrm{Sn}$ & 1,88 & 4,29 \\
$\mathrm{Si}$ & - & 0,77 \\
\hline
\end{tabular}

(insolubles entre sí) (Fig. 10). Tanto la capa de hierro como la de bronce quedan interrelacionadas con una capa, denominada B, formada por una mezcla de las dos aleaciones (Fig. 11), donde la matriz está formada por hierro como componente mayoritario, mientras que en el límite de grano 


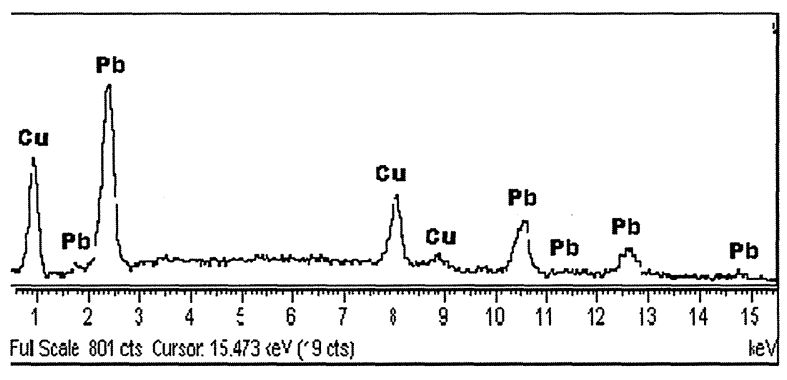

Figura 10. Análisis EDS de la zona D marcada en la figura 7.

Figure 10. EDX Identification of the zone $D$ composition in the figure 7 .

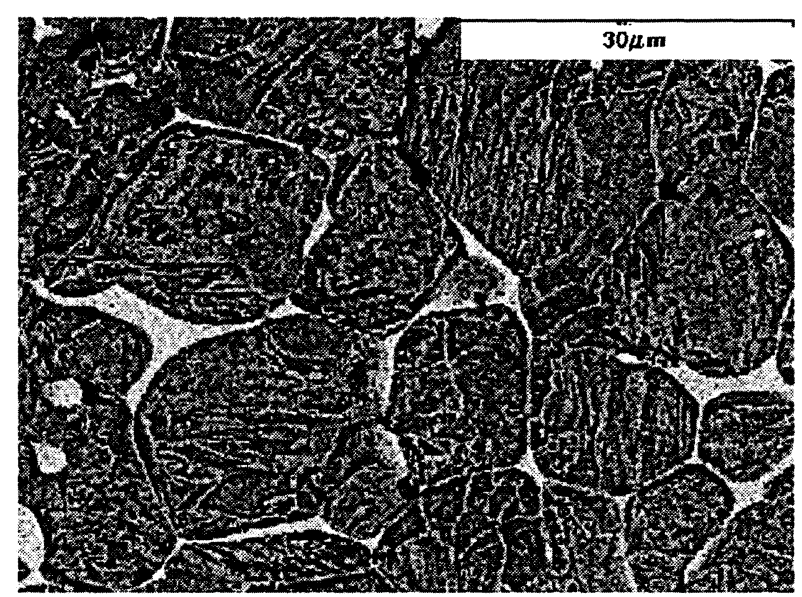

Figura 11. Detalle de figura 7 en la zona denominada como B.

Figure 11. Detail of the figure 7. Zone B.

queda segregado el cobre, que se combina con estaño, junto con trazas de silicio (Tabla IV).

Tabla IV. Composiciones de las fases presentes en la zona B de la figura 7

Table IV. Composition weight, \% of the phases in the zone $B$ of the figure 7

\begin{tabular}{crc}
\hline \multirow{2}{*}{ Elemento } & \multicolumn{2}{c}{ \% En Peso } \\
\cline { 2 - 3 } & Matriz & Límite de grano \\
\hline $\mathrm{Fe}$ & 90,28 & 8,73 \\
$\mathrm{Cu}$ & 9,72 & 82,95 \\
$\mathrm{Sn}$ & - & 7,57 \\
$\mathrm{Si}$ & - & 0,75 \\
\hline
\end{tabular}

Los valores de dureza medidos en esta pieza indican medidas muy dispares. Así se presentan valores de las fases de bronce de $90 \mathrm{HV}$, valores en las láminas de hierro de $75 \mathrm{HV}$ y zonas transformadas de $119 \mathrm{HV}$. Por otra parte, los valores de dureza obtenidos en el badajo son de $69 \mathrm{HV}$.

\section{CONCLUSIONES}

El método de fabricación utilizado en esta pieza deducido a partir del análisis radiológico nos causa contradicciones para dar una explicación al método de fabricación de esta pieza, ya que justifica tanto la posibilidad de fabricación a partir del plegado de una única lámina de hierro y soldadura lateral en una de sus caras, como la fabricación a partir de dos láminas plegadas y soldadura en ambas caras laterales del cencerro.

El equivoco queda aclarado tras el estudio microestructural que revela que la soldadura longitudinal que se aprecia en la radiografía no es tal y se trata del badajo.

\section{REFERENCIAS}

[1] P. PAlol, La Villa romana de La Olmeda de Pedrosa de la Vega (Palencia), Ed. Excma. Diputación provincial de Palencia, Palencia, España, 1993.

[2] J. CoRTES, La necrópolis de La Olmeda, Ed. Departamento de Cultura. Diputación provincial de Palencia, Palencia, España, 1990.

[3] R.F. TYLECOTE, The early history of metallurgy in Europe, Ed. Longman, London and New York, UK and USA, 1987.

[4] S. RoviRA, Manuel Fernández-Miranda y la arqueometalurgia española, Ed. Diputación de Albacete, Albacete, España, 1996.

[5] R. Pleiner, Proc. Coloquio Internacional de Minería y metalurgia en las antiguas civilizaciones mediterráneas y europeas, vol. I, Madrid, España, Ed. Ministerio de Cultura, 1989, pp.137-140.

[6] R.F. TyLECOTE, Furnaces, crucibles and slags, The coming of the Age of Iron, Ed, New Haven and London Yale University press, London, UK, 1980, pp. 208-221.

[7] J.M. Gómez de Salazar y A. Soria, Rev. Metal. Madrid 36 (2000) 118-125. 